\title{
Challenges and Solutions for Organizational Design in Urban Digitalization
}

\author{
Máté Szilárd Csukás ${ }^{1}$, Viktor Bukovszki², András Reith ${ }^{3}$
}

\begin{abstract}
Digitalization in cities - often branded as smart city (SC) transition - carry the potential for highly inclusive, evidence-based decision making in urban planning, responding to the increasing pressures cities face. However, investments have thus far been slower to deliver the expected impacts. Thus, the attention of the discourse is turning towards organizational structures addressing complexity, scalability, and procedural challenges of SC transition. Given such turn has regime-challenging implications, there is a need for practice-based research in the niches of SC transition, supporting policymaking inductively. This study outlines the barriers inherent in conventional organizational models (public sector, private-supplier, and academic-professional) to SC transition, and makes a case for alternative models. The barriers are retrieved through an extensive literature review, and a series of focus groups with key stakeholders involved in SC transition, and processed as a design problem for a new organizational model. The final design is a nested platform model based on open innovation and a lean approach to urban planning. The paper concludes with a proof of concept to overcome organizational barriers, validated by the stakeholder focus groups.
\end{abstract}

Keywords: urban planning, platform, open innovation, assessment, smart city, organizational models

\section{Introduction}

The increasing rate of population growth and concentration has resulted in mounting demands in resources in cities amidst a global effort to sustainably meet these demands, mitigating and adapting to environmental, economic and social pressures (United Nations Human Settlements Programme, 2009). Many cities in the EU seek to cope with these challenges through a smart city (SC) approach to urban planning and management (UPM), referring to the uptake of cutting edge ICT (mostly data-driven) solutions to inform and integrate problem-solving in urban processes (Manville et al., 2014; Ruhlandt, 2018).

Although such digitalization in cities carries the potential for highly inclusive, evidence-based decision making in UPM, investments have thus far been slow to deliver the expected impacts (Barns, 2018). Various frameworks, methodologies, and supporting tools have been developed by academia, the public sector, and suppliers to support SC implementation, each with their specific limitations. Supplier-driven frameworks are too narrow in scope, focusing on urban subsystems relatable to their products and services (Robinson, 2015), (Barns, 2018). City-based and some academic frameworks are too specific to a type of city (Manville et al., 2014; Shen, Jorge Ochoa, Shah, \& Zhang, 2011); or to a discipline (Bibri \& Krogstie, 2017a); while broader frameworks are too generic 
and abstract (Klopp \& Petretta, 2016). The utilization of urban data itself is limited to indicator listing, without analytic and decision-support functionalities for UPM (Mori \& Christodoulou, 2012; Nordregio, 2004). On the other hand, the mismatch of a fast-paced SC market supply and a slow-to-react demand in UPM is driving cities into ad-hoc investments. It is therefore important to investigate why SC frameworks fail to enable transition on the cities side.

One possible explanation for the lack of progress is the cross-cutting nature of its challenges. SC transition challenges can be classified as problems of sociotechnical scalability, the complexity of the subject matter, and procedural difficulties (Dezi, Pisano, Pironti, \& Papa, 2018; C. Lim, Kim, \& Maglio, 2018a; Nel, du Plessis, \& Landman, 2018; Offenhuber \& Schechtner, 2018; Reed, 2008; Vanolo, 2014; Zhang et al., 2017). Each of these axes combines social and technical aspects. First, sociotechnical scalability refers to challenges rooted in the addition of more and more people, organizations, information, and hardware in UPM. On the one hand, various data producing and processing systems and actuators need to be interoperable, and the data models integrated (C. Lim, Kim, \& Maglio, 2018b).

On the other hand, it is challenging to identify, address, empower stakeholders, and incentivize them to participate in a multi-actor decision-making process (Offenhuber \& Schechtner, 2018). It is not apparent which information will be crucial for whom (Reed, 2008), how knowledge is diffused (Dezi et al., 2018), how security is ensured (Zhang et al., 2017) and how the power asymmetries in delicate social structures translate to a more digitalized UPM (Vanolo, 2014). Second, complexity challenges stem from cities being multidimensional complex adaptive systems with deeply interconnected subsystems and interdependent actors (Nel et al., 2018), which is difficult to formalize in a digital environment. Moreover, practical SC implementation must overcome a standardization - customization problem: the breadth of contextual information used in UPM inhibits the replicability and mainstreaming of approaches to practical SC implementation (Manville et al., 2014). Finally, procedural difficulties refer to unpreparedness in the planning process: failure to link urban data to users (Badii et al., 2017), lack of methods to consider city goals and outcomes (Yigitcanlar et al., 2018), a lack of data-based services (Aguilera, Peña, Belmonte, \& López-de-Ipiña, 2017), and lack of tools supporting planning (Mora, Deakin, \& Reid, 2018).

Scalability, complexity, and procedural challenges cannot be solved through technological innovation alone. Indeed the attention of SC discourse is turning towards organizational structures to deliver transition (Ruhlandt, 2018). Academia, supplier, and public sector governed SC frameworks are sharing one particular attribute: they were initiated, sponsored and maintained and used by single or few entities, even if the development itself was a result of a collaborative effort. The goal of this study is to make a case for an alternative organizational composition based on collaborative networks that are better suited to deliver SC transition. More specifically, the goal of this study is to answer: are there barriers inherent in the conventional organizational models meeting the challenges of SC transition? If so, do an alternative, collaborative network models exist to overcome these barriers? 


\section{Methodology}

SmartCEPS (Smart City Evaluation Platform and Services) is an ongoing research work, intending to develop and roll out a data-driven, scalable, evidence-based, participatory decision-support system for UPM in small and medium-sized cities. It consists of (1) an urban data analytics toolbox, (2) a methodology to use urban data analytics in multi-actor UPM, (3) a virtual marketplace of ICT solutions with urban applications. SmartCEPS is a multi-stakeholder service actively involving municipalities, technology suppliers, citizens, sectoral urban specialists, and generalist consultants - with the business model being training and supplying these generalist consultants. This paper presents the management, particularly the organizational challenges of an application of an innovative data-driven urban planning service and methodology that involves the orchestration of a wide range of urban stakeholders.

The research has three distinct phases. As a first step, an extensive literature review was undertaken to identify challenges of SC transition, regarding (1) multistakeholder ecosystems, (2) data-driven applications, (3) knowledge-intensive innovative services, and (4) urban planning. Key ideas were revealed during an inductive category development procedure (Mayring, 2000). After processing about $50 \%$ of selected papers, revision of categories and pre-validation of findings begun in the next phase. Three separate focus group sessions were conducted, with a selected group of experts, from the three most essential stakeholder categories: city managers, urban planning professionals, and technology suppliers. These events aimed to validate, check the reliability, and formulate new categories of organizational challenges. They were semi-structured to allow the identification of additional concepts. Next, information gathered in these events was processed, parallel with the remaining literature, and findings were incorporated into the category development task.

After the categories were finalized, organizational barriers were distributed to their respective stakeholder groups, which served as a problem space for designing the organizational framework of SmartCEPS. Finally, the design decisions in the corresponding solution space were validated in follow-up focus groups, and the proposed organizational concept has been revised accordingly.

\section{Results}

Organizational barriers manifest differently among the three main stakeholders involved: (1) public, (2) private and (3) professional, while some (4) general barriers are not unique to any of them. Table 1 summarizes the organizational barriers to SC transition.

\subsection{Public organizational barriers}

In this paper, we partly adopt the stakeholder classification of Ruhlandt (Ruhlandt, 2018), including public, private, academic, and civic. Public stakeholders are described as "institutions", "public subject", "government agencies", "political leadership and administrative proponents" in the literature. Category development process 
identified that public organizational barriers fall into the following groups: Management, Power \& influence, Networks, and Innovation.

In governance, the capacity to adapt and integrate is mentioned to be a critical barrier to the SC transition. Given the volatility of the SC market and urban pressures in general, the need for dynamic control mechanisms is accentuated (O'Reilly \& Tushman, 2008). The current practice in Europe is characterized by weak convergence and integration mechanism among interventions, a failure to realize the added value offered by coordination of resources and from the joint efforts of stakeholders (Praharaj, Han, \& Hawken, 2018). Both barriers are connected to an authoritative governance approach which fails to enable knowledge, resource, and responsibility pooling (Sørensen \& Torfing, 2018). According to interviewed city managers, such approach also results in trading evidence-based planning with political struggles when resources are constrained, which leads to inertia against any public innovation both in the leadership and down the chain of command. The lack of collaborative governance and planning is seen as a critical obstacle to an SC-ready level of adaptive and integrative capacity.

Incapable organizational structures hinder the SC transition. Despite the high relevance, there were participants from all stakeholder groups, who expressed concerns about the viability of their respective organizational background, to be able to address all the needs of SC transition. Especially the 'old' structure of city governance that operates in these "byzantine labyrinth" of silos, requires cross-organizational management. Roles and responsibilities must be clearly defined in the case of external and internal transactions as well. Again, a non-technological, but management and planning problem was identified as a problem for the transition. Without cross-departmental governance, collaboration with various stakeholders cannot be guaranteed (Whyte et al., 2014). It is also a vital issue, mentioned by city managers, that their processes are slow and inefficient, due to the lack of inter-departmental connections.

Furthermore, SC project and developments usually realized in a multicollaborative (open) environments, where the roles and responsibilities of city managers are challenging to determine (Angelidou, 2014). These difficulties can include their position or even their authority. Some practitioners from technology provider companies mentioned that in many cases, negotiations fail because they do not meet with the right person who is qualified to understand the subject matter but also has the authority to make decisions. In other cases, city managers are afraid to decide on specific issues because of a risk aversive culture inherent in the public sector (Sørensen \& Torfing, 2018).

The threat to power and influence is also an often-mentioned factor across different groups. It is also a general hindering factor for organizations that face changes and resist them. Some stakeholders or departments might play a more prominent role in the transition, while others' are excluded or oppressed. Participants perceive that there are beneficiaries but also disadvantages, which can cause stress in and in-between organizations. That is an excellent reason to adopt an inclusive and participative SC design. Otherwise, the particular power dynamics of longstanding stakeholders are invoked, causing tensions in the city, inevitably hindering SC development.

Inability to properly engage stakeholders is a distinct category of organizational barriers. It is the responsibility of SC developers to design and implement meaningful 
public-private partnerships, which will function as a sustainable ecosystem (Vanolo, 2014). They have to take the role of stakeholder leadership, because they are the most credible actor, with legitimacy. Engagement of relevant ecosystem participants is a very challenging task because they must be provided with viable incentives to stimulate them. In most SC investments and development processes, practitioners feel as if there are always important actors who are not at the table. There is a knowledge gap among relevant actors. This collaborative thinking is also real for the co-initiation of participatory processes (Daniell et al., 2010). If SC transition is not co-initiated from the bottom-up, it is met with resistance or indifference (Sørensen \& Torfing, 2018). SC success is very vulnerable to the degree of public engagement. The knowledge gaps above have spillover effects on the collaboration as well. Beside others, civic engagement is of the highest importance in SC development. Despite its perceived importance, citizen involvement is still not an integral part of the practice. There are several platformbased ICT solutions for this challenge - i.e., Citizen Relationship Management (CRM), Knowledge Hub, but currently, they are not appropriately informed, have no agency in decision-making, and treated mostly as sources of information at best (crowdsensing).

Another critical category in public services is connected to innovation. One significant capability of public actors in SC transition is to combine the innovativeness of a diverse group of stakeholders, through partnerships. The resulting interactions will be the source of creativity and novel solutions (Anttiroiko, 2016). Urban openness degree enables a user (citizen)-driven innovation in existing and new services. Similarly, to governance barriers, there is an utmost need for multi-stakeholder management. Table 1 below collects the identified public barriers.

Table 1: Public Organizational Barriers (Source: own edition)

\begin{tabular}{|c|c|c|c|}
\hline \multicolumn{4}{|c|}{ Public Organizational Barriers } \\
\hline Management & Power \& influence & Networks & Innovation \\
\hline $\begin{array}{l}\text {-Lack of } \\
\text { coordination } \\
\text { mechanisms } \\
\text {-Authoritative } \\
\text { strategic } \\
\text { approaches } \\
\text {-Lack of horizontal } \\
\text { integration } \\
\text { (functional } \\
\text { silos) }\end{array}$ & $\begin{array}{l}\text { - Conflict of } \\
\text { interests } \\
\text { - Power } \\
\text { asymmetries } \\
\text { - Resistance to } \\
\text { change }\end{array}$ & $\begin{array}{l}\text {-Underperforming } \\
\text { collaborative } \\
\text { design } \\
\text { - Insufficient } \\
\text { collaborative } \\
\text { capabilities } \\
\text {-Lack of co- } \\
\text { initiation }\end{array}$ & $\begin{array}{l}\text {-No mutual learning } \\
\text { and knowledge } \\
\text { diffusion practices } \\
\text {-Lack of innovation } \\
\text { capabilities } \\
\text {-Closed organizational } \\
\text { design } \\
\text { - Inadequate learning } \\
\text { from peer cities }\end{array}$ \\
\hline
\end{tabular}

\subsection{Private organizational barriers}

Private organizations are mostly described as firms and enterprises, but also industry, or market (Ruhlandt, 2018). Many 'general' organizational barriers identified in the case of public and professional actors are also recognizable in this group. It is challenging for companies to align their value-creating processes into the SC transition 
context. As mentioned before, the immaturity and complexity of the concept force ventures to focus on particular domains of SC within their preexisting competencies, they while they lack a holistic view, and the capacity to thrive with such a contextdependent value offering as smart cities. Both the literature and the city manager focus group identified technology push from companies (Angelidou, 2015). Today, cities and technology providers do not speak a common language regarding technology and social outcomes of SC developments. There is a massive gap between vision and implementations, causing stress between the two actors (Bibri, 2018).

Furthermore, sales processes are not efficient because of different organizational structures. Private organizations - despite the high expectations - are still exploring the market, accumulating investments, and expect high growth in the future. Vendors claim that advanced and sophisticated technologies are inadequate to gain user satisfaction in realistic SC environments. In an unstandardized market, where individual cases require a high-level specification, successful implementation of tech solutions is very challenging, particularly the design, optimization, and requirement checks.

Another significant organizational barrier is related to collaboration, knowledge diffusion, and innovation. Competitive strategies of the private sector result in wasted resources on rivalry and conflicts, but which is more critical, limited exchange of knowledge (Sørensen \& Torfing, 2018). Taking into account the complexity of urban challenges and requirements, the absence of mutual learning jeopardize the highly desired innovation capabilities. Ad-hoc approaches are present in case of private actors as well. They deploy technologies and solutions that become available with technological advancement, but without an adequately demonstrated customer need and value proposition. Table 2 below sums up the identified private organizational barriers.

Table 2: Private Organizational Barriers (Source: own edition)

\begin{tabular}{|l|l|}
\hline \multicolumn{2}{|c|}{ Private Organizational Barriers } \\
\hline \multicolumn{1}{|c|}{ Competitive drive } & \multicolumn{1}{c|}{ Innovation \& Technology } \\
\hline $\begin{array}{l}\text { - Product focus over needs } \\
\text {-Biased assessments }\end{array}$ & $\begin{array}{l}\text {-Closed innovation process } \\
\text {-Applicability over real needs }\end{array}$ \\
\hline
\end{tabular}

Distortions between technology push and application pull results in a mismatch between supply and demand. The imbalance is partly because of the difficulty to articulate SC expectations in terms of product specification (Bukovszki, Apró, Khoja, Essig, \& Reith, 2019). Suppliers also find it challenging to create new products and services for specific city needs, as they are more inclined to work with their existing portfolios. They experience considerable tension between standardization and customization because there are trade-offs between these two categories: cities require solutions that are entirely based on their local particularities, but firms want to commercialize efficiently scalable products. In the case of private and professional groups, there is an 'oversupply' because supplier driven SC frameworks are designed around the products and services offered by the issuing companies. 


\subsection{Professional organizational barriers}

Private organizations are mostly described as universities and research bodies in the literature (Ruhlandt, 2018). Practitioners - i.e., consultants may also be treated in this category. Many organizational barriers of professional actors are related to knowledge diffusion and capabilities. Until now, there is no common understanding of the SC concept, which makes the interpretation and practical intervention challenging for firms. Results of the focus group with professionals also confirm this status, showing that SC is a fuzzy concept; everyone sees a different meaning in it. The interpretation of smart cities today is a capability that requires immersive knowledge. Otherwise, the adverse effects - i.e., technology dependence, exclusion of social impacts will prevail (Yigitcanlar et al., 2018). UPM is interdisciplinary, and SC frameworks either represent a single dimension, field, discipline, or they become too vague when trying to be holistic (Bibri \& Krogstie, 2017b; Sabatini-Marques, da Costa, Chang, Yigitcanlar, \& Selig, 2018). Furthermore, SC development is still treated as an outcome, not as a planning process. Table 3 below collects the identified professional organizational barriers.

Table 3: Professional Organizational Barriers (Source: own edition)

\begin{tabular}{|c|c|c|}
\hline \multicolumn{3}{|c|}{ Professional Organizational Barriers } \\
\hline Dissension & Collaboration & Operational \\
\hline $\begin{array}{l}\text { - Biased assessments Disciplinary } \\
\text { complexity \& immaturity } \\
\text {-Fragmented knowledge } \\
\text { generation } \\
\text { - Biased interpretations }\end{array}$ & $\begin{array}{l}\text {-Closed innovation } \\
\text { process } \\
\text {-Applicability over real } \\
\text { needs }\end{array}$ & $\begin{array}{l}\text { - Lack of actionable outputs } \\
\text { - Missing practice-oriented } \\
\text { qualifications } \\
\text { - Focus only on outcomes }\end{array}$ \\
\hline
\end{tabular}

Similarly, to public organizational barriers, resistant to change is also a phenomenon among professional stakeholders' group. During the focus group session, they were conflicted with data-driven planning approach, opposing their current practice. In academic publications, there is a myriad of SC indicator systems. Problem is with their practical usability, the difficulty of transposing theory to practice. They provide good descriptions, but no executable programs (Mori \& Christodoulou, 2012). This challenge is an organizational barrier because relevant actors work independently. Horizontal action among practitioners is desired. Focus group participant also expressed that SC frameworks choose between offering comparability and accuracy, since adding more and more contextual information is needed during the planning process (standardization and customization conundrum) (Bukovszki et al., 2019). Another issue is ad-hoc approaches, which is closely related to the lack of inter-organizational collaboration and meaningful engagement of stakeholders. Ad-hoc approaches make it risky to correctly identify and select interventions that can deliver the desired complex socio-spatial outcomes. Table 4 below summarizes the identified generic barriers.

Table 4: Generic Organizational Barriers (Source: own edition) 


\begin{tabular}{|c|c|}
\hline \multicolumn{2}{|c|}{ Generic Organizational Barriers } \\
\hline Management & Cultural \\
\hline $\begin{array}{l}\text {-Too large number heterogeneous of actors } \\
\text {-Interdependencies (system complexity) } \\
\text {-Communication channels (information flow) }\end{array}$ & $\begin{array}{l}\text {-Divergent value perspectives } \\
\text {-Different frames of reference } \\
\text {-Different mindset }\end{array}$ \\
\hline
\end{tabular}

\section{Discussion of a Proposed Model: Urban Planning Platform}

In this chapter, we are going to describe our proposed model and how it can address the previously identified organizational barriers. The diversity of stakeholders and technological solutions require new models of cooperation to parallel regulate urban actors by governance but provide an innovative, evolving environment at the same time. The authors argue, that the SC transition has a fundamentally a disruptive nature, transforming existing markets and conventional organizational or business structures, which will eventually create new public- and technology management models. In such an environment, the knowledge-intensive digital transformation of cities requires open innovation models - with particular attention to the inflows and outflows of knowledge (Chesbrough \& Brunswicker, 2014) - because of the challenges posed by the unique urban features, multi-collaborative environments, and immaturity of the market. The proposed model is a platform that facilitates an open innovation network, with a Community of Innovation (CoI) (M. Lim \& Ong, 2019) and Technology Matchmaking mechanism. The adoption of platform thinking to urbanism and particularly smart cities is getting more and more attention in the literature, as a solution for participatory innovation (Anttiroiko, 2016), mediating public-private partnerships (van der Graaf \& Ballon, 2019), or delivering services (Rajakallio, Cuthbertson, Pulkka, \& Junnila, 2018). Platforms contend the linearity of existing SC developments, facilitating space for interdependencies, increasingly dominating the urban landscape. Platform-based ecosystems, as demonstrated in the, e.g., high tech industry that they are capable of orchestrating a diverse group of innovative actors towards a common goal with a set of rules and incentives, surpassing the traditional organizational boundaries (Gawer \& Cusumano, 2014). The SmartCEPS urban planning platform incorporates the design and value creation logic of platforms.

\subsection{Platform attributes}

The SmartCEPS platform architecture is nested, comprising of two layers. The first layer would function for UPM. It is a discourse in a network of stakeholders, municipal admin, specialist professionals, solution providers, data-producing infrastructure, and peer cities. Orchestration and leadership of the ecosystem would be carried out by a certified consultant network, with a lean project management approach, meaning with early and persistent user-oversight minimizing waste. The second layer would be an urban information system, continuously developed by a discourse in a network of urban professionals. This layer would be a cooperative platform, which is managed and improved by the SmartCEPS consultant community through the 
platform's infrastructure. This second layer hosts content creation and curation for the analytic tools supporting multi-actor collaboration on the first layer, which would enable a procedure where new technological solutions and concepts evolve from bottom-up collaborations among various stakeholders. Monitoring of urban performance and correcting deviations from urban planning goals makes it possible for a city-level cybernetic control (Carver \& Scheier, 2012). The figure 1 below illustrates the layers of the platform architecture.

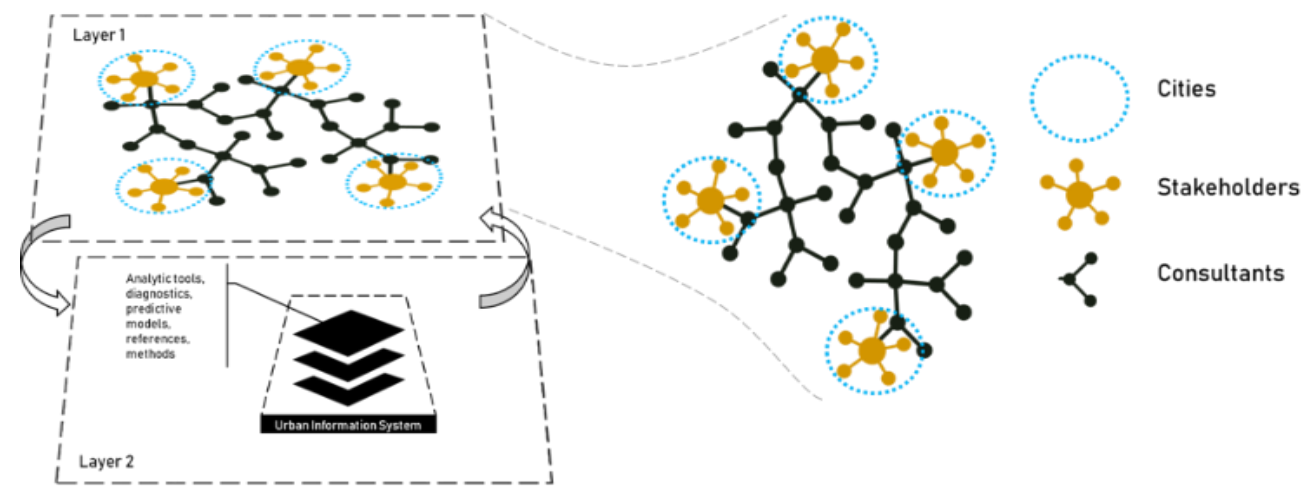

\section{Figure: SmarCEPS Platform Layers (Source: own edition)}

The first layer facilitates the core interaction of transacting urban performance data that is generated by the city assessment framework. Key actors are consultants, a community of certified professionals, city managers, who initiate joining the platform, civic community, participating in whole of the process and technology suppliers, who contribute to the materialization of the proposed solutions. Knowledge is exchanged among actors to collaboratively (1) diagnose city problems and define strategic priorities (2) propose and select policy and urban development (3) invest in SC applications of ICT solutions. The principal value proposition of the platform is solving the organizational barriers by translating different actor frames of reference to a standardized data model and vice versa. As this value depends on the accuracy of underlying models, the SmartCEPS consultants are incentivized to actively contribute to the second layer, which in this sense is an open innovation platform. More accurate diagnostic, predictive models, references, increasingly sophisticated methods to transfer contextual information to global models boost both the individual and collective value of the consultants. Figure 2 below is intended to explain the multi-collaborative and multi-level characteristic of the proposed model. 


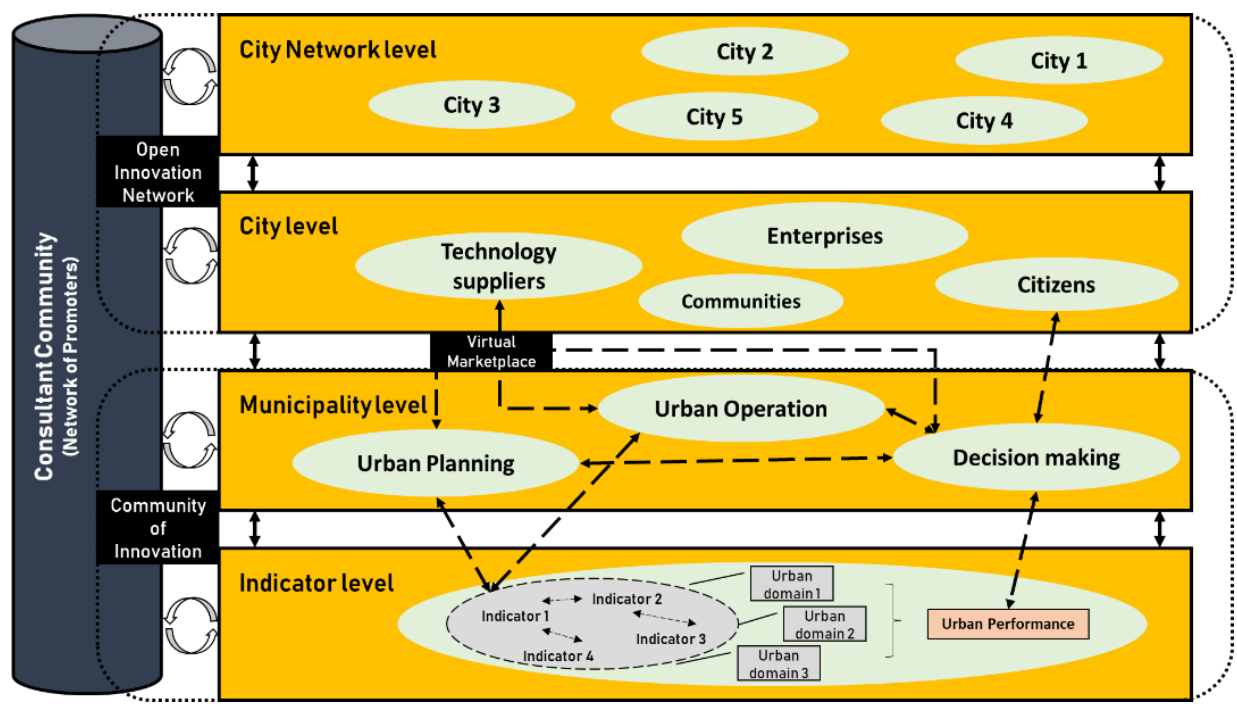

2. Figure: High-level platform architecture with stakeholder relations (Source: own edition)

SmartCEPS Urban Planning Platform adopts conditions for value creation in the following way:

Modularity: the proposed model consists of self-functional components that are separately upgradable, contributing to the adaptability of the system. Modules are the (a) Consultancy network, which is a community of certified SC professionals, who are bounded by shared frames of references; (b) Virtual Marketplace that is an expanding network of technology provider firms; (c) urban analytics toolset, which facilitates a common 'language' to communicate urban problems in-between multiple stakeholders, and to be evolved by the community.

Customizable standardization: the platform is using common standards to assess cities, formulate action plans, and initiate action in practice, based on the analytics and translation of urban performance data. However, the more in-depth data content is built up iteratively by UPM stakeholders during the planning process, ensuring customizability.

Complementarity: complementarity manifests in both actions and technical systems as well. First, consultants work together with public administration and professionals to design interventions that are co-initiated and validated by citizens, which are realized by private companies. Second, from the technical system point of view, city assessments provide each city an 'algorithm', a set of data that define the city, which translates to the Virtual Marketplace in the form of interventions.

Connectivity: coordination and management of each module and the stakeholders are assigned to city dedicated consultants who act as 'conductors', or community managers of the urban planning ecosystem. Inputs and outputs are all shared and accessible by users on the SmartCEPS platform infrastructure. System components are connected in an integrative way. 


\subsection{Overcoming organizational barriers}

Public organizational barriers: The ecosystem creation is in the heart of the proposed model, where new channels are established, through a common language to cooperatively initiate solutions, implement existing products/services and capitalize on the competences in the local ecosystem. The consultants provide management in the process, following municipality goals. They take multiple roles - e.g., promoting the network, managing the community, enabling knowledge flows. They act as conductors of the discourse, encompassing specialists, municipal managers, civic communities, and other stakeholders. Consultants are trained to use and interpret data and analytics to evolve and expand this discourse. UPM platform layer establishes new connections in the departmental and stakeholder network. Responsibilities are assigned in the design process, and consultants provide leadership, capacities, and bridge knowledge gaps. The platform facilitates the creation and accessibility of continuous feedback from the consulting community on the interactions, synergies, contextual factors, impacts of urban development and policy recommendations, in a shared urban performance architecture, which ensures the integrative nature of the platform. Figure 3 visualizes the identified challenges and the offered solutions of the platform in the public sector.

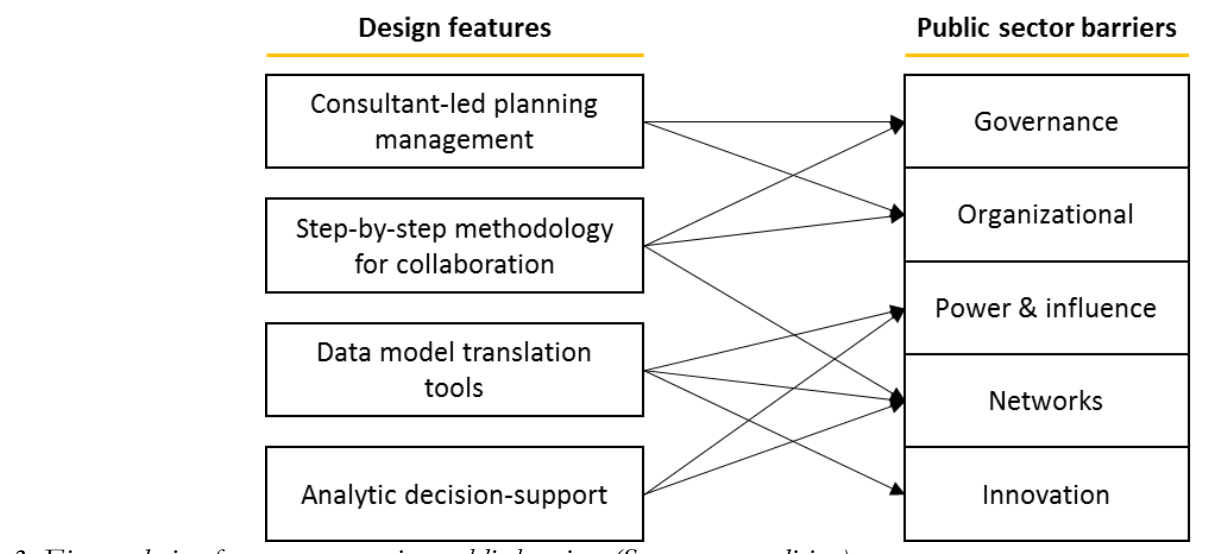

3. Figure: design features overcoming public barriers (Source: own edition)

Collaboration and co-initiation are ensured by step-by-step methodology, identifying crucial multi-stakeholder decision points, modules to outline stakeholder impacts and to involve relevant stakeholders. Through the information system reference projects are linked to context, goals, city performance. They are described with a universal language and shared underlying data. Latent city relationships - i.e., similarity, complementarity can be defined because of continuous monitoring with a shared reference frame and underlying data, operated by a network of consultant professionals.

Private organizational barriers: the Virtual Marketplace (VM) module of the platform is intended to resolve the tension between the technology push and application pull forces, providing a convenient, yet efficient forum for companies to register their products or solutions, directly linked to the potential impact on the cities' algorithms meaning the quantified effect of the solutions. Mediation creates matchmaking between cities and companies, in a way that the platform translates problems and needs into 
solution (innovation) requests, decomposing them into specific impact measures. These indicators transcribe to specific technical descriptions, disseminating requests through the network. VM enables a more accurate presentation of products and services in terms of urban performance, while the consultant network specifies city needs in term of technology solutions. Figure 4 illustrates the identified challenges and the offered solutions of the platform in the private sector.

Design features

Private sector barriers

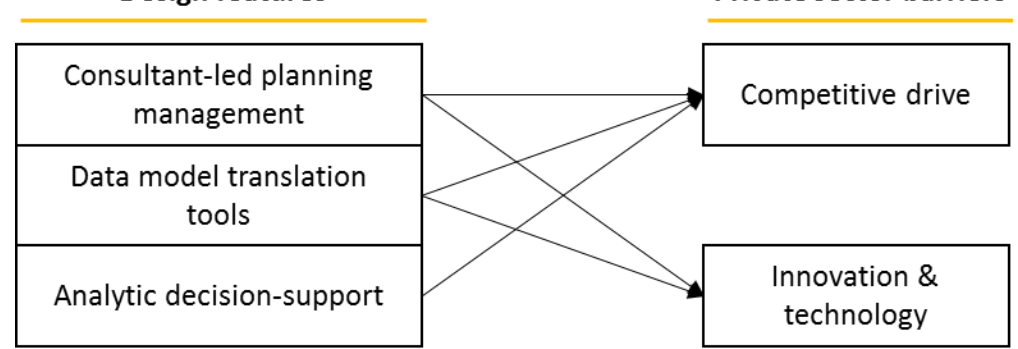

4. Figure: design features overcoming private barriers (Source: own edition)

Professional barriers: the SmartCEPS platform is centred on urban performance, translated to different actors' perspectives by common standards and modules. Discourse is not running on obscure conceptualizations and inauthentic approaches, but on performance-based analytics that increases transparency. Platform modules support data analytics in an open innovation framework, continuously peerreviewed, updated, and developed by a wide range of dedicated professionals. Mutual learning incentivized by providing an integrative and scalable, yet city-specific service model, that is globally transferable. The urban information system module is gradually customized during the planning process from global standards maintained by a community of consultants. Protocols to integrate consultant feedback into different layers of standards - i.e., global, context-bound, city-specific. Figure 5 illustrates the identified challenges and the offered solutions of the platform in the professionalacademic sector.

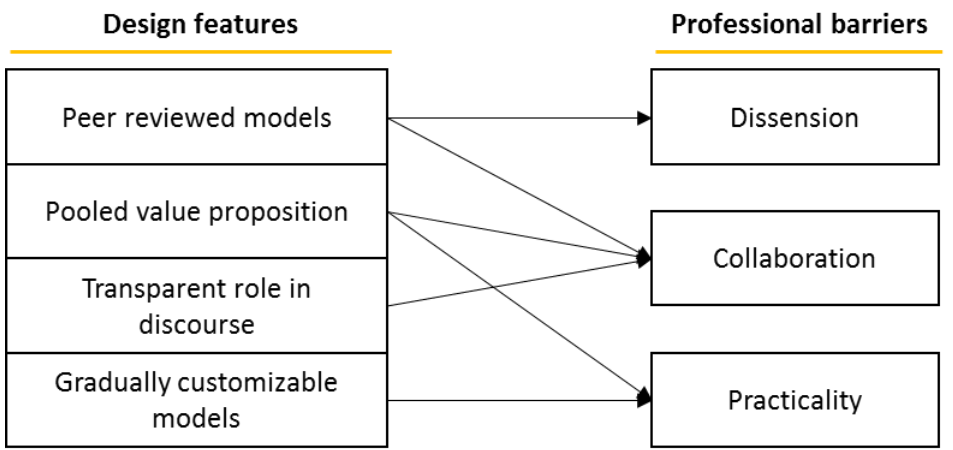

\section{Figure: design features overcoming professional barriers (Source: own edition)}

Generic barriers: VM module address interoperability issues, and in the planning process, additional costs of possible incompatibilities are taken into account. Different levels of the assessment provide flexibility in a data-scarce and information 
divided environment. Missing or insufficient values are imputed. The dedicated consultants individually address the peculiarities of the local data environment. Organizational and cultural differences are aligned by using urban data and analytics to support inter-stakeholder discourse, which is the core competence of SmartCEPS consultants. Methodologies and tools to translate among frames of reference are added to the system and are continuously developed by the consultants. Figure 6 illustrates the identified challenges and the offered solutions that are non-specific to any target groups.

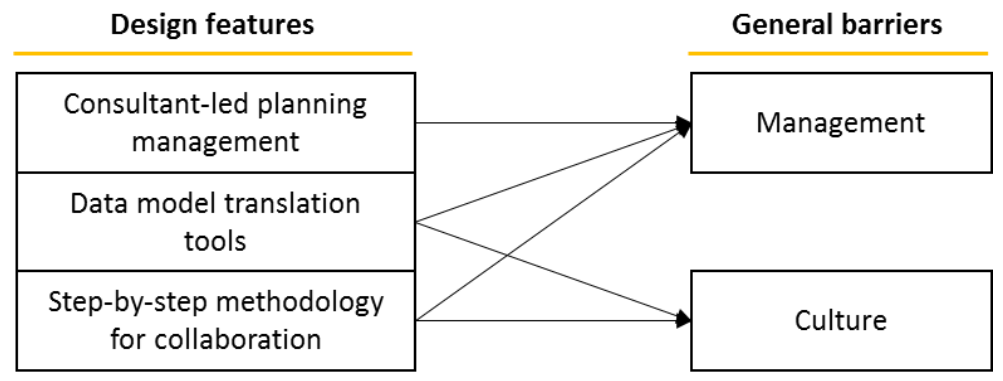

\section{Figure: design features overcoming generic barriers (Source: own edition)}

\section{Conclusion}

The platform framework is shown to be a viable organizational model for SC transition because such a transformation is reliant on long-term, cross-cutting, and crosssectoral collaboration. In the design study, this is achieved by positioning SC transition as a continuous self-reinforcing protocol for the day-to-day process of UPM.

SmartCEPS is a nested platform for collaborative planning on the first layer, and the continuous evolution of underlying methods and models on the second layer. The backbone of the first layer is the translation of stakeholder frames of reference to a standardized, open-ended, dynamic model of urban performance, which allows for more transparent participation at lower transaction costs. The second layer creates a plus-sum game to continuously evolve and adapt the supporting tools for UPM, by pooling the value proposition of professionals while rewarding active contributors, thus for the first layer. It is notable, however, that the SmartCEPS design is limited in addressing increased data security needs (due to more interactions), it has a high entry barrier for many stakeholders to participate, and is disruptive to conventional deliberative processes - all of which are consequences of a collaborative UPM process.

The SmartCEPS project will continue with demonstrating its design in real-life experiments, but further research will be needed to expand on the trade-offs and minimal entry criteria of a platform-based UPM.

\section{Acknowledgements}

The SmartCEPS project has received funding from the European Union's Eurostars-2 joint programme with co-funding from European Union Horizon 2020 research and innovation programme under reference no. E! 10243/14/Q. Project no. NEMZ_15-1-2016-0028 has been implemented with the support provided from the 
National Research, Development and Innovation Fund of Hungary, financed under the NEMZ-15 funding scheme.

\section{References}

Aguilera, U., Peña, O., Belmonte, O., \& López-de-Ipiña, D. (2017). Citizen-centric data services for smarter cities. Future Generation Computer Systems, 76, 234-247. https://doi.org/10.1016/j.future.2016.10.031

Angelidou, M. (2014). Smart city policies: A spatial approach. Cities, 41, S3-S11. https://doi.org/10.1016/j.cities.2014.06.007

Angelidou, M. (2015). Smart cities: A conjuncture of four forces. Cities, 47, 95-106. https://doi.org/10.1016/j.cities.2015.05.004

Anttiroiko, A. V. (2016). City-as-a-platform: The rise of participatory innovation platforms in finnish cities. Sustainability (Switzerland) (Vol. 8). https://doi.org/10.3390/su8090922

Badii, C., Bellini, P., Cenni, D., DIfino, A., Paolucci, M., \& Nesi, P. (2017). User Engagement Engine for Smart City Strategies. 2017 IEEE International Conference on Smart Computing, SMARTCOMP 2017. https://doi.org/10.1109/SMARTCOMP.2017.7947059

Barns, S. (2018). Smart cities and urban data platforms: Designing interfaces for smart governance. City, Culture and Society, 12(December 2016), 5-12. https://doi.org/10.1016/j.ccs.2017.09.006

Bibri, S. E. (2018). Smart Sustainable Cities of the Future. Cham: Springer International Publishing. https://doi.org/10.1007/978-3-319-73981-6

Bibri, S. E., \& Krogstie, J. (2017a). Smart sustainable cities of the future: An extensive interdisciplinary literature review. Sustainable Cities and Society, 31, 183-212. https://doi.org/10.1016/j.scs.2017.02.016

Bukovszki, V., Apró, D., Khoja, A., Essig, N., \& Reith, A. (2019). From assessment to implementation: design considerations for scalable decision-support solutions in sustainable urban development. In Central Europe towards Sustainable Building. Prague: Unpublished.

Carver, C. S., \& Scheier, M. F. (2012). Cybernetic Control Processes and the Self-Regulation of Behavior. The Oxford Handbook of Human Motivation, (May 2018), 1-29. https://doi.org/10.1093/oxfordhb/9780195399820.013.0003

Chesbrough, H., \& Brunswicker, S. (2014). A Fad or a Phenomenon? The Adoption of Open Innovation Practices in Large Firms. Research-Technology Management, (March-April), 16-25. https://doi.org/10.5437/08956308X5702196

Daniell, K. A., White, I., Ferrand, N., Ribarova, I. S., Coad, P., Rougier, J., ... Burn, S. (2010). Coengineering Participatory Water Management Processes : Theory and. Ecology And Society, 15(4), $1-$ 38.

Dezi, L., Pisano, P., Pironti, M., \& Papa, A. (2018). Unpacking open innovation neighborhoods: le milieu of the lean smart city. Management Decision, 56(6), 1247-1270. https://doi.org/10.1108/MD-04-20170407

Gawer, A., \& Cusumano, M. A. (2014). Industry Platforms and Ecosystem Innovation. Journal of Product Innovation Management, 31(3), 417-433. https://doi.org/10.1111/jpim.12105

Klopp, J. M., \& Petretta, D. (2016). Can we actually agree on indicators to measure urban development?

Lim, C., Kim, K.-J., \& Maglio, P. P. (2018a). Smart cities with big data: Reference models, challenges, and considerations. Cities, 82, 86-99. https://doi.org/10.1016/j.cities.2018.04.011

Lim, M., \& Ong, B. Y. (2019). Communities of innovation. International Journal of Innovation Science, IJIS-092017-0086. https://doi.org/10.1108/IJIS-09-2017-0086

Manville, C., Cochrane, G., Cave, J., Millard, J., Pederson, J. K., Thaarup, R. K., ... Kotternik, B. Mapping Smart Cities in the EU (2014). European Union. https://doi.org/10.1017/CBO9781107415324.004

Mayring, P. (2000). Qualitative Content Analysis Philipp Mayring 3 . Basic Ideas of Content Analysis. In Forum Qualitative Sozialforschung (Vol. 1, p. 10).

Mora, L., Deakin, M., \& Reid, A. (2018). Smart-City Development Paths: Insights from the First Two Decades of Research. In Green Energy and Technology (Vol. 8, pp. 403-427). Elsevier. https://doi.org/10.1007/978-3-319-75774-2_28 
Mori, K., \& Christodoulou, A. (2012). Review of sustainability indices and indicators: Towards a new City Sustainability Index (CSI). Environmental Impact Assessment Review, 32(1), 94-106. https://doi.org/10.1016/j.eiar.2011.06.001

Nel, D., du Plessis, C., \& Landman, K. (2018). Planning for dynamic cities: introducing a framework to understand urban change from a complex adaptive systems approach. International Planning Studies, 23(3), 250-263. https://doi.org/10.1080/13563475.2018.1439370

Nordregio. Potentials for polycentric development in Europe Co-financed by the European Community through the Interreg III ESPON (2004). Stockholm.

O’Reilly, C. A., \& Tushman, M. L. (2008). Ambidexterity as a dynamic capability: Resolving the innovator's dilemma. Research in Organizational Behavior, 28, 185-206.

https://doi.org/10.1016/j.riob.2008.06.002

Offenhuber, D., \& Schechtner, K. (2018). Improstructure - an improvisational perspective on smart infrastructure governance. Cities, 72, 329-338. https://doi.org/https://doi.org/10.1016/j.cities.2017.09.017

Praharaj, S., Han, J. H., \& Hawken, S. (2018). Towards the right model of smart city governance in India. International Journal of Sustainable Development and Planning, 13(2), 171-186. https://doi.org/10.2495/SDP-V13-N2-171-186

Rajakallio, K., Cuthbertson, R., Pulkka, L., \& Junnila, S. (2018). Creating urban platforms — Opportunities and challenges for innovation in commercial real estate development. Cities, 77, 92-103. https://doi.org/https://doi.org/10.1016/j.cities.2018.01.016

Reed, M. S. (2008). Stakeholder participation for environmental management: A literature review. Biological Conservation, 141(10), 2417-2431. https://doi.org/10.1016/j.biocon.2008.07.014

Robinson, R. (2015). 6 inconvenient truths about Smart Cities. Retrieved from http://theurbantechnologist.com/2015/02/15/6-inconvenient-truths-about-smart-cities/

Ruhlandt, R. W. S. (2018). The governance of smart cities: A systematic literature review. Cities, 81(October 2017), 1-23. https://doi.org/10.1016/j.cities.2018.02.014

Sabatini-Marques, J., da Costa, E. M., Chang, D. L., Yigitcanlar, T., \& Selig, P. M. (2018). Knowledge-based, smart and sustainable cities: a provocation for a conceptual framework. Journal of Open Innovation: Technology, Market, and Complexity, 4(1). https://doi.org/10.1186/s40852-018-0087-2

Shen, L. Y., Jorge Ochoa, J., Shah, M. N., \& Zhang, X. (2011). The application of urban sustainability indicators - A comparison between various practices. Habitat International, 35(1), 17-29. https://doi.org/10.1016/j.habitatint.2010.03.006

Sørensen, E., \& Torfing, J. (2018). Co-initiation of Collaborative Innovation in Urban Spaces. Urban Affairs Review, 54(2), 388-418. https://doi.org/10.1177/1078087416651936

United Nations Human Settlements Programme. (2009). Planning Sustainable Cities: Global Report on Human Settlements 2009. (N. D. Mutizwa-Mangiza, Ed.). London: Earthscan/ UN-HABITAT. https://doi.org/https://doi.org/10.1002/psp.616

v. d. Graaf, S., \& Ballon, P. (2019). Navigating platform urbanism. Technological Forecasting and Social Change, 142(December 2017), 364-372. https://doi.org/10.1016/j.techfore.2018.07.027

Vanolo, A. (2014). Smartmentality: The Smart City as Disciplinary Strategy. Urban Studies, 51(5), 883-898. https://doi.org/10.1177/0042098013494427

Whyte, J., Hart, T., Yanrong, K., Lei, Z., Cai, C., Yuming, G., ... Ying, C. (2014). EU-China Smart and Green City Cooperation "Comparative Study of Smart Cities in Europe and China " - White Paper - prepared for Ministry of Industry and Information Technology ( MIIT ) DG CNECT, EU Commission with China Academy of Telecommunications Res, (March).

Yigitcanlar, T., Kamruzzaman, M., Buys, L., Ioppolo, G., Sabatini-Marques, J., da Costa, E. M., \& Yun, J. J. (2018). Understanding 'smart cities': Intertwining development drivers with desired outcomes in a multidimensional framework. Cities, 81, 145-160. https://doi.org/https://doi.org/10.1016/j.cities.2018.04.003

Zhang, K., Ni, J., Yang, K., Liang, X., Ren, J., \& Shen, X. S. (2017). Security and Privacy in Smart City Applications: Challenges and Solutions. IEEE Communications Magarine, 55(1), 122-129.

https://doi.org/10.1109/MCOM.2017.1600267CM 